\title{
Winteröffnung des Lukmanierpasses mit organisatorischen Massnahmen
}

\author{
Nora Zürcher-Gasser \\ Christian Wilhelm \\ Fabrizio Conceprio \\ Stefan Margreth
}

\author{
Gadola AG $(\mathrm{CH})^{*}$ \\ Amt für Wald und Naturgefahren Graubünden $(\mathrm{CH})$ \\ Lucchini-Mariotta e Associati SA (CH) \\ WSL-Institut für Schnee- und Lawinenforschung $(\mathrm{CH})$
}

\begin{abstract}
Winter opening of the Lukmanier Pass, and accompanying organisational measures
\end{abstract}

\begin{abstract}
On an experimental basis, the Lukmanier Pass has been open during ten winter seasons since winter 2000/2001, through the efforts of the Pro Lucmagn association. In 2010, a request was made to keep the pass open on a permanent basis, which led to a revision of the safety process and a re-evaluation of the risks. Overall, there has been a more significant reduction of risks than assumed initially, thanks to measures to close the road and trig. ger avalanches artificially. The estimated residual risks are at an acceptable level. Nevertheless, despite the chosen organisational measures there remain significant risks, which originate mostly from uncertainty connected with human judgement. Looking back, it appears that the risk analysis of 1998 was appropriate, and adequate to estimate in advance the trial operation of the winter opening of the Lukmanier Pass.
\end{abstract}

Keywords: risk analysis, Lukmanier Pass, winter opening, organisational measures doi: $10.3188 /$ szf.2014.0292

*CH-7172 Rabius, E-Mail zuercher.gasser@gadola-ag.ch er Lukmanierpass (1915 $\mathrm{m}$ ü. M.) verbindet die Surselvà (GR) mit dem Bleniotal (TI). Auf einer Strecke von rund $20 \mathrm{~km}$ wird die Passstrasse durch 84 Lawinenzüge bedroht (Abbildung 1). Um diese Gefährdungen zu reduzieren, wurden schon vor Jahrzehnten sowohl auf der Süd-als auch auf der Nordseite zahlreiche Galerien, Ablenkđämme und andere Schutzbauten erstellt, welche allerdings nur einen Teil des Gefährdungspotenziales abdecken. Bis im Jahr 2000 war deshalb der Pass im Winter geschlossen.

In den späten 1990er-Jahren wurde der Verein Pro Lucmagn gegründet mit dem Ziel, eine Winteröffnung des Lukmanierpasses zur Toûrismusförderung in der Surselva und im Bleniotal zu erreichen. Im Auftrag des Kantons Graubünden analysierte das WSL-Institut für Schnee- und Lawinenforschung (SLF 1998) die mit einer Winteröffnung zu erwartenden Risiken und zeigte verschiedene Varianten zu deren Reduktion auf. Aus diesen Untersuchungen resultierte, dass eine Winteröffnung des Lukmanierpasses ohne zusätzliche bauliche Schutzmassnahmen und nur mit den organisatorischen Massnahmen «Sperren» und «künstliche Lawinenauslösung» nicht empfohlen werden kann. Nach umfangreichen weiteren Abklärungen und der Erarbeitung eines nur auf organisatorischen Massnahmen beruhenden Sicherungskonzeptes waren die Kantone Graubün-

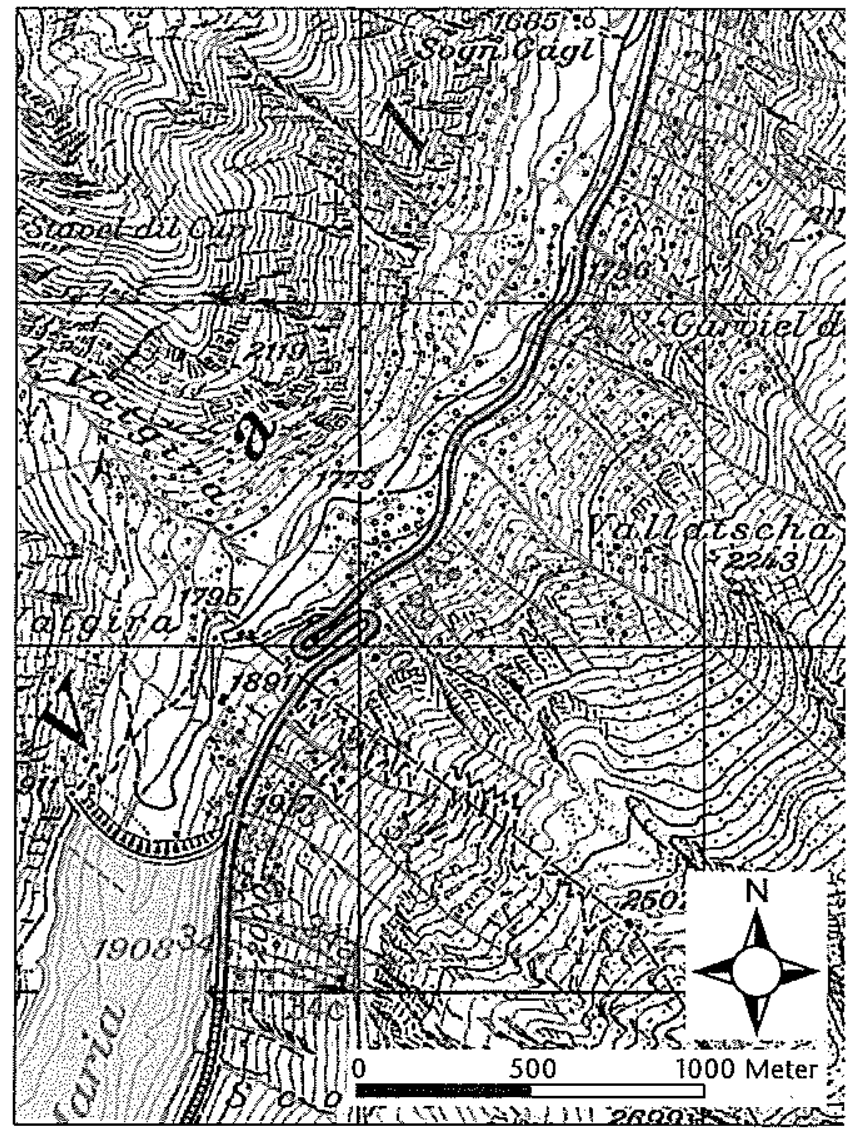

Abb 1 Gehöufte Lawinenzüge nördlich der Scopi-Galerie. oswisstopo 




Abb 2 Starke Schneeverwehungen oder Eisbildung können nebst der Lawinengefahr dazu führen, dass die Passstrasse geschlossen werden muss.

den und Tessin trotzdem bereit, eine Versuchsphase für die Passöffnung von fünf Jalıren zu bewilligen. Bereits der erste, äusserst schneereiche Winter 2000/2001 stellte eine grosse Herausforderung dar und erforderte viele Sperrtage. Dank grossem Einsatz der Sicherungsverantwortlichen konnte der Pass in der ersten sowie in einer zweiten fünfjährigen Versuchsphase erfolgreich offen gehalten werden.

Seit dem Winter 2009/2010 ist die Winteröffnung unbefristet bewilligt. Diese unbefristete Bewilligung war für die Kantone Graubünden und Tessin sowie für den Verein Pro Lucmagn Anlass, die Organisation der Winteröffnung zu überprüfen und $a b-$ zuklären, ob allenfalls die Verfügbarkeit der Passstrasse im Winter weiter verbessert werden kann.

\section{Ausgangslage und Fragestellungen}

Risikoanalysen zur Beurteilung von Naturgefahren waren Ende 1990er-Jahre in der Praxis noch wenig verbreitet. Obwohl die Methoden entwickelt waren (BUWAL 1999, Wilhelm 1999) und die Nationale Plattform Naturgefahren den Paradigmenwechsel von der Gefahrenabwehr zur Risikokultur postuliert hatte (PLANAT 1998), dauerte es weitere zehn Jahre bis das Risikokonzept zur Beurteilung aller Naturgefahren entwickelt war (Bründl 2009) und grossflächige Risikoanalysen durchgeführt wurden (z.B. ASTRA 2012). In seinem Gutachten hat das SLF mit einer Risikoanalyse verschiedene Varianten einer Winteröffnung des Lukmanierpasses quantitativ beurteilt (SLF 1998).

Der Lawinenwinter 1998/1999 stellte den Lawinenschutz in den Alpen auf die Bewährungsprobe. Die permanenten Massnahmen erwiesen sich als grunđsätzlich geeignet. Als verbesserungsfähig stufte man die organisatorischen Massnahmen ein; sie wurden aber als vergleichsweise kostengünstige Massnahmen mit noch grossem Potenzial für den Verkehrsachsenschutz postuliert (SLF 2000). In diesem Umfeld war es eine grosse Herausforderung, ten Lukmanierpass ab dem Winter 2000/2001 mit organisatorischen Massnahmen zu öffnen.

Bei der Überprüfung des heutigen Konzepts der Winteröffnung im Jahr 2011 (Conceprio \& Gasser 2011) wurden die folgenden Fragen diskutiert:

- Wurden die Lawinenrisiken beim Lukmanierpass 1998 adäquat eingeschätzt?

- Wie effizient hat der Sicherungsdienst im Zeitraum von 2000 bis 2010 gearbeitet?

- Haben die organisatorischen Massnahmen die erhoffte Wirkung bezüglich Risikoverminderung und erhöhter Verfügbarkeit gezeigt?

- Wo liegen die Grenzen des bisher verwendeten Sicherungskonzeptes?

Zur Beantwortung dieser Fragen muss die Wirkung von Sperrtagen auf die Risikoverminderung quantitativ erfasst werden können. Die von Wilhelm (1999) entwickelte Methodik wurde von Margreth et al (2003) für verschiedene Passstrassen angewendet und von Rheinberger et al (2009) hinsichtlich Nutzenbewertung weiterentwickelt. Die Nutzenbewertung wie auch die Verfügbarkeit respektive die Kosten von Strassensperrungen werden heute immer wichtiger (ASTRA 2012).

Der vorliegende Artikel erläutert nach folgend das Sicherungskonzept zur Winteröffnung des Lukmanierpasses und zeigt die Resultate von zehn Jahren Betrieb. Insbesondere wird die Wirkung der Strassensperrung und der künstlichen Lawinenauslösung auf die Risikoverminderung beurteilt. Dazu wird die vom SLF 1998 a priori durchgeführte Risikoanalyse mit der im Jahr 2011 durch die Ingenieurbüros Lucchini-Mariotta e Associati SA und Gadola AG (Conceprio \& Gasser 2011) a posteriori durchgeführten Risikoanalyse verglichen.

\section{Winteröffnung $a b$ dem Winter 2000/2001}

\section{Sicherungskonzept}

Das Sicherungskonzept, für welches der Verein Pro Lucmagn verantwortlich ist, beruht in erster Linie auf Strassensperrungen; zusätzlich werden auf der Nordseite des Passes Lawinen künstlich ausgelöst. Eine technische Kommission mit je einem Vertreter der Nord- und der Südseite sowie der Sicherungsdienst mit je einem Verantwortlichen für die Nord- und die Südseite sind für das Operative zuständig. Die jährlichen Betriebskosten beliefen sich zwischen den Wintern 2000/2001 und 2009/2010 durchschnittlich auf CHF $580000 .-$.

Der Sicherungsdienst beurteilt täglich, ob der Pass offen sein kann oder geschlossen werden muss. 


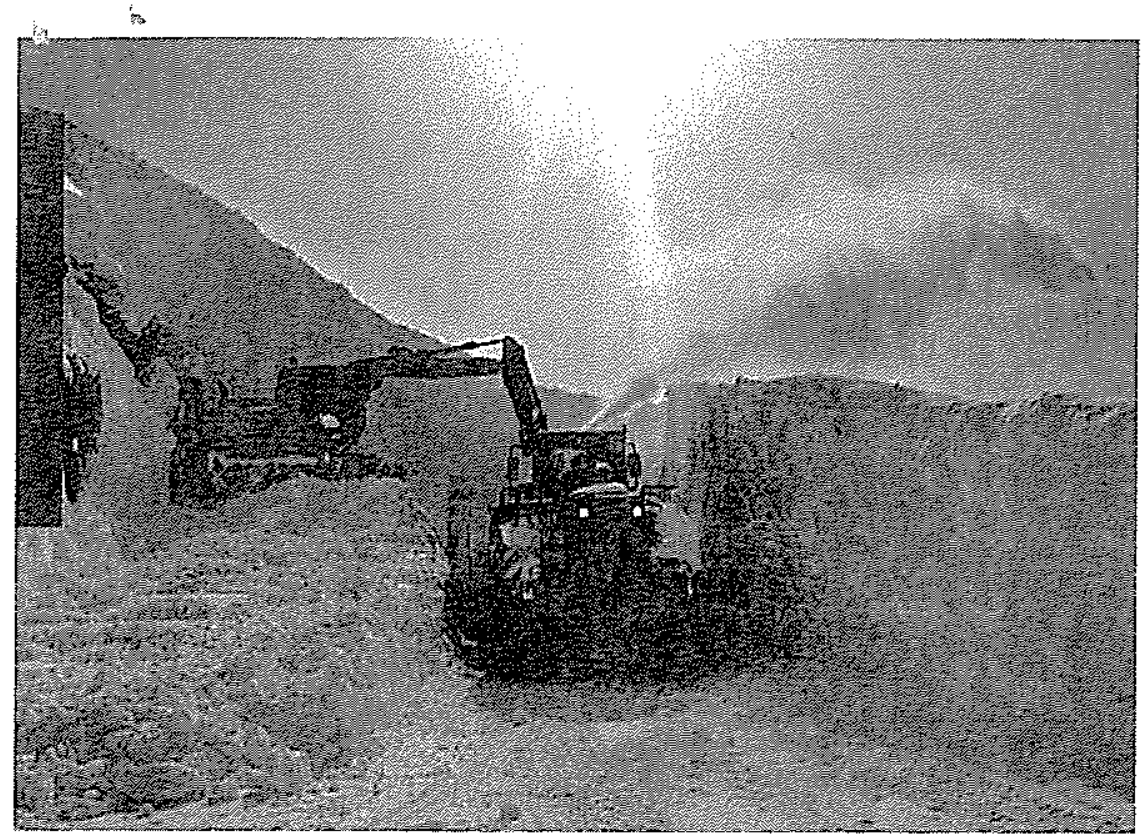

Abb 3 Die Schneeräumung bedingt insbesondere nach grossen Lawinenabgängen einen erheblichen Maschinen- und Zeitaufwand. Räumungsfahrzeug im Lawinenzug Nr. 34. Foto: O. Flepp, Pro Lucmagn
Nebst der Sperrung des Passes werden auf der Nordseite zusätzlich Lawinensprengungen aus dem Helikopter vorgenommen. Auf der Südseite werden wegen gefährdeter Gebäude und Waldflächen sowie aus Naturschutzgründen keine Lawinensprengungen durchgeführt. Sämtliche Lawinen, sowohl spontane wie künstlich ausgelöste, werden im Lawinenkataster mit Kartierung der Umrisse und Angaben zu Verschüttungsbreite und -höhe der Strasse erfasst. Wird die Situation als lawinensicher eingeschätzt, bietet der Sicherungsdienst bei Bedarf die Schneeräumungsequipe auf (Abbildung 3). Der aktuelle Strassenzustand wird über verschiedene Kanäle (Polizei, Internetseite, Info-Telefon, SMS- und E-MailDienst) veröffentlicht.

\section{Erfahrungen mit der Winteröffnung}

Die Wintermonate November bis April umfassen 181 Tage, die als durchschnittliche potenzielle Winterzeit für den Lukmanierpass bezeichnet werden können. Von 1967 bis 2000 war der Pass im Winter geschlossen, was durchschnittlich an 155 Tagen pro Winter der Fall war, wobei die Unterschiede zwischen den einzelnen Wintern beträchtlich sind. Mit der Winteröffnung konnten die Tage, an denen der Pass ganztägig geschlossen war, von durchschnittlich 155 auf 37 gesenkt werden (Abbildung 4).

Ausschlaggebend für Risikoberechnungen sind nicht nur diejenigen Tage, an welchen der Pass während 24 Stunden geschlossen ist, sondern zusätzlich auch die Sperrstunden der Nachtsperre (18.00-08.00) und Nachmittagssperre (Frühlingssituation, 11.00-19.00/21.00). Diese Sperrstunden wurden auf Norm-Sperrtage umgerechnet, wobei ein Norm-Sperrtag 24 Stunden entspricht (wird im

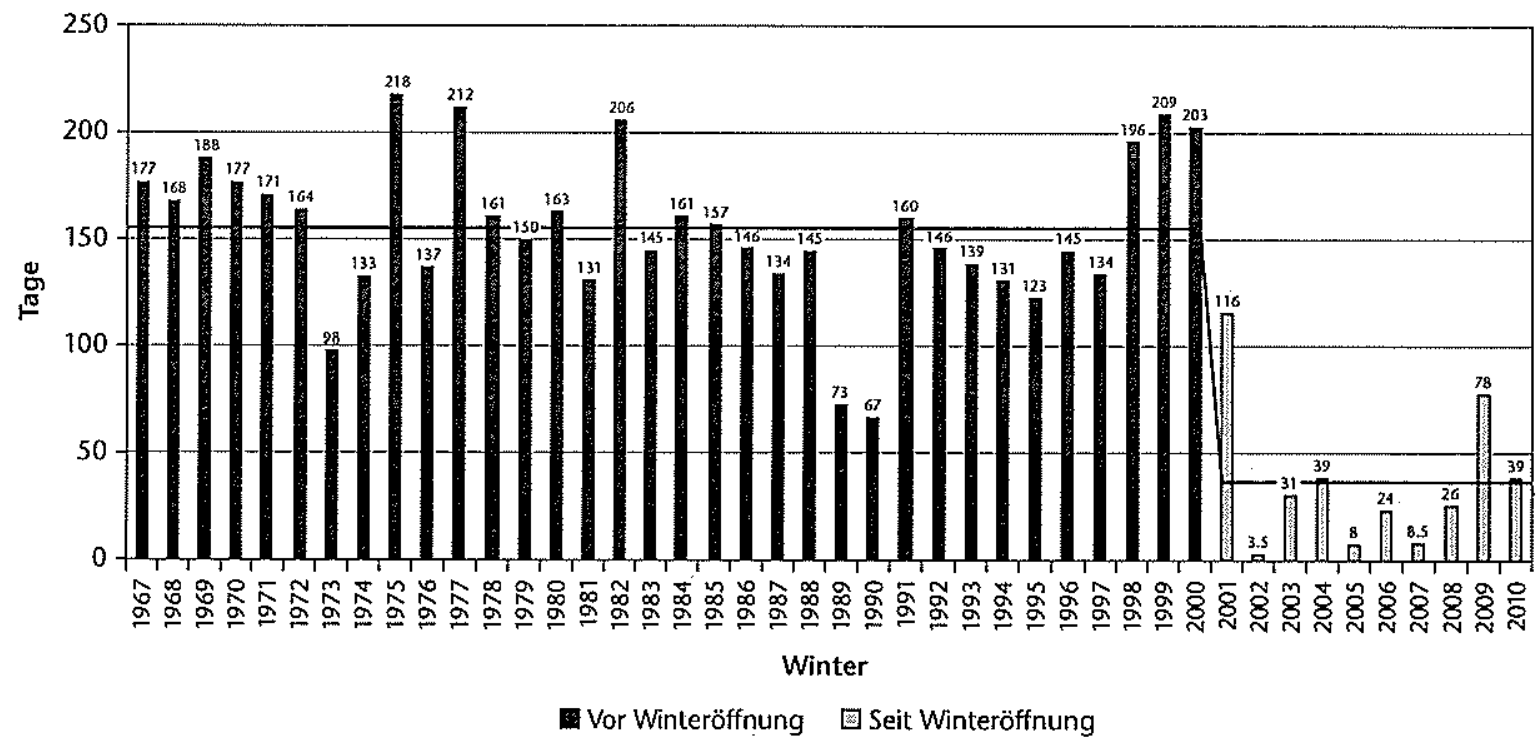

Abb 4 Anzahl Tage, an denen der Lukmanierpass während der Winterperiode (November bis April) ganztags geschlossen war (ab Winter 2000/2001 mit Winteröffnung). 1967 ist als Winter 1966/1967'zu interpretieren. Horizontale Linien: durchschnittliche Anzahl Schliesstage (1966/1967-1999/2000: 155 Tage, 2000/2001-2009/2010: 37 Tage). Auffallend ist, dass die Variabilität sowohl vor als auch nach 2000/2001 sehr gross war. Dies zeigt die starke Abhängigkeit der Passöffnung von den jeweiligen Witterungsverhältnissen. Quelle: Conceprio \& Gasser 2011. 


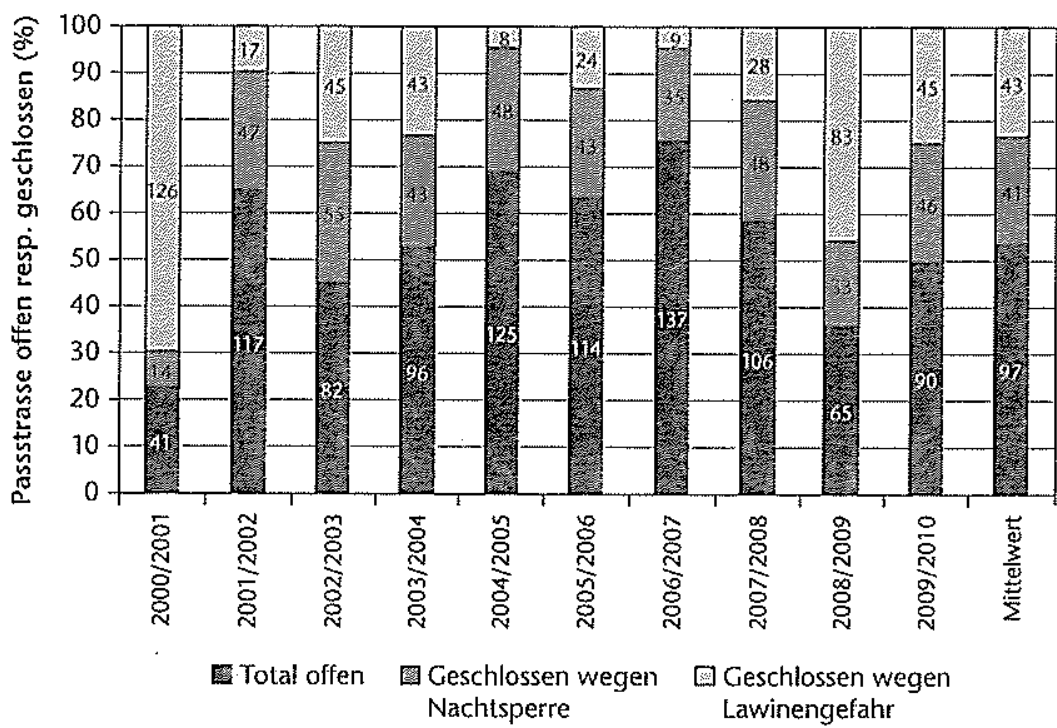

Abb 5 Anzahl und Anteil der Tage, an denen die Passstrasse offen respektive geschlossen war, wobei einzelne Stunden auf Normtage $(24 \mathrm{~h})$ umgerechnet wurden. $100 \%$ entsprechen 181 respektive 182 Wintertagen (jeweils van Anfang November bis Ende April).

Folgenden als Sperrtag bezeichnet). Ein Tag mit der Öffnungszeit 08.00-18.00 ging also mit 10 Stunden offen und 14 Stunden gesperrt und somit als 0.58 Sperrtage in die Berechnungen ein. Zudem ist für die Risikoberechnungen zu berücksichtigen, dass nicht nur wegen Lawinengefahr gesperrt wird, sondern auch wegen Schneeverwehungen und Eisbildung (Abbildung 2). Weiter werden auch aus Kostengründen Nachtsperren verhängt.

Von 2000 bis 2010 waren durchschnittlich 84 Sperrtage zu verzeichnen (Abbildung 5). Minimal waren 44 Sperrtage notwendig (2006/2007) und maximal 140 Sperrtage (Winter 2000/2001). Werden nur die Sperrtage infolge Lawinengefahr berücksichtigt, ergeben sich minimal 8 Sperrtage (Winter 2004/2005 und 2006/2007) und maximal 126 Sperrtage (Winter 2000/2001). Durchschnittlich war die Passstrasse infolge Lawinengefahr an 43 Tagen gespert, wovon an 37 Tagen ganztägig. Bemerkenswert ist, dass rund die Hälfte der Sperrtage durch ungünstige Witterungsverhältnisse (z.B. Schneeverwehungen oder Eisbildung; Abbildung 2) verursacht werden.

Weiter ist für die Risikoberechnungen der durchschnittliche tägliche Verkehr im Winter (DTV) von Bedeutung. Dazu wird die Zählstation von Cam- pra verwendet. Pro Winter ergaben sich zwischen knapp 32000 und gut 52000 Fahrzeugbewegungen, was einem DTV von 409 Fahrzeugen entspricht (Tabelle 1).

Die Fahrzeugbewegungen korrelieren recht gut mit der Anzahl Tage, an denen die Passstrasse geöffnet war: Je grösser die Verfügbarkeit ist, desto mehr Verkehr wird generiert (Tabelle 1). Die Ausnahme im Winter 2009/2010 dürfte auf spezielle Witterungsbedingungen zurückzuführen sein. Mit durchschnittlich 97 Öffnungstagen an insgesamt 181 Wintertagen waren knapp 40000 Fahrzeugbewegungen pro Winter zu verzeichnen.

Die Vermutung, dass der DTV auch mit der Anzahl geöffneter Tage korrelieren könnte, wird mit den Daten der letzten zehn Jahre nicht bestätigt (Tabelle 1). So war zum Beispiel im Winter 2008/2009 der Pass sehr oft gesperrt, und trotzdem (oder deshalb?) ist in diesem Winter der höchste DTV zu verzeichnen. Vermutlich kommt hier eher die Konzentration des Verkehrs auf die wenigen geöffneten Tage zum Ausdruck.

\section{Beurteilung der Wirkung des Sicherungskonzepts}

Mit der unbefristeten Bewilligung für die Winteröffnung im Jahr 2009 stellte sich die Frage, wie wirkungsvoll das gewählte Konzept ist und wie es allenfalls verbessert werden kann. Um diese Fragen zu beantworten, gaben die beiden Kantone in Jahr 2010 eine Studie in Auftrag, um die Risikoanalyse des SLF aus dem Jahr 1998 (SLF 1998) anhand der in den Jahren 2000/2001 bis 2009/2010 gewonnenen Daten zu überprüfen respektive die Risiken neu zu beurteilen (Conceprio \& Gasser 2011).

\section{Datengrundlage}

Im Jahr 1998 standen dem SLF nur lückenhafte Daten zur Lawinenaktivität zur Verfügung, zudem musste der DTV geschätzt werden. Die Lawinenaktivität wurde insbesondere durch Geländebeurteilungen und Erfahrungen mit anderen Passstrassen abgeschätzt. Mit der Passöffnung im Winter 2000/2001 wurden die beobachteten Lawinenab-

\begin{tabular}{|c|c|c|c|c|c|c|c|c|c|c|c|}
\hline $\begin{array}{l}\text { Fahrzeug gewe } \\
\text { gungen }\end{array}$ & $2000 / 2001$ & $2001 / 2002$ & $2002 / 2003$ & $2003 / 2004$ & $2004 / 2005$ & $2005 / 2006$ & $2006 / 2007$ & $2007 / 2008$ & $2008 / 2009$ & $2009 / 2010$ & $\begin{array}{l}\text { Mittel- } \\
\text { wert }\end{array}$ \\
\hline Richtung Sud & \multirow{3}{*}{$\begin{array}{l}\text { Keine Daten } \\
\text { vorhanden }\end{array}$} & 24393 & 16754 & 14501 & 21017 & 21486 & \multirow{3}{*}{$\begin{array}{l}\text { Keine Daten } \\
\text { vorhanden }\end{array}$} & 19717 & 16980 & 20188 & 19380 \\
\hline Richtung Nord & & 27652 & 9654 & 17891 & 28123 & 22416 & & 1846 & 14919 & 20420 & 20605 \\
\hline Total & & 52045 & 36408 & 32392 & 44440 & 43902 & & 38180 & 37899 & 40608 & 39984 \\
\hline $\begin{array}{l}\text { Anzahl Tage } \\
\text { offen (Normtage) }\end{array}$ & 41 & 17 & 82 & 96 & 125 & 14 & 37 & 106 & 65 & 90 & 97 \\
\hline DTV & & 444 & 447 & 338 & 355 & 384 & & 361 & 488 & 452 & 409 \\
\hline
\end{tabular}

Tab 1 Anzahl Fahrzeugbewegungen (Richtung Süd, Richtung Nord und Total) auf der Lukmanierpassstrasse (November bis April) für die Jahre 2000 bis 2010. Der mittlere durchschnittliche tägliche Verkehr (DTV) wurde mit der Anzahl Normtage berechnet, die alle Sperrstunden respektive Sperrtage pro Winter umfassen. 
gänge auf die gesperrte respektive offene Passstrasse, das Verkehrsaufkommen, die Öffnungs- und Sperrzeiten sowie die Resultate der künstlichen Lawinenauslösung detailliert erfasst. Im Vergleich zu 1998 bestand für die neue Risikoanalyse eine umfassende Datenlage.

\section{Methode: Risikoanalyse}

Die Risikoanalyse wurde wie bereits 1998 gemäss Wilhelm (1999) durchgeführt. Dadurch können die Ausgangsrisiken direkt miteinander verglichen werden. Dies ist insbesondere deshalb interessant, weil 1998 nur lückenhafte Daten zur Verfügung standen und heute ein umfassender Datensatz der wichtigsten Eingangsgrössen vorliegt. Die Methode beinhaltet die nachfolgenden Schritte:

\section{Berechnung des Ausgangsrisikos}

Im Wesentlichen wird anhand der Grössen "Wiederkehrdauer der Lawine», "Verschüttungsbreite der Lawine», «durchschnittlicher täglicher Verkehr (DTV)" und "Fahrzeuggeschwindigkeit» die Wahrscheinlichkeit abgeschätzt, mit welcher Personen auf der Strasse ohne Sicherungsmassnahmen, d.h. ohne Sperrungen und künstliche Lawinenauslösung, durch Lawinen zu Schaden kommen könnten.

\section{Bestimmung der erforderlichen Anzah/ Sperrtage} für 100-prozentige Risikoreduktion

Anhand der Gefahrenstufe im lokalen Lawinenbulletin (erstellt durch den Sicherungsdienst) wurde abgeschätzt, mit wie vielen Sperrtagen das Risiko von Schadenfällen durch Lawinen $\mathrm{zu}$ «100\%» hätte reduziert werden können. Berücksichtigt wurden gemäss Wilhelm (1999) alle Tage mit der Gefahrenstufe $\geq 3$ (erheblich), sowie die Hälfte der Tage mit der Gefahrenstufe 2 (mässig).
Herleitung der theoretisch optimalen

\section{Risikoverminderungsfunktion}

Mithilfe des Lawinenkatasters wurde für jeden Winter ermittelt, an welchen Tagen wie viele Strassenverschüttungen stattfanden. Daraus konnte das durch diese Strassenverschüttungen entstandene Risiko (in Todesfällen pro Jahr) berechnet werden, falls die Strasse an diesem Tag geöffnet gewesen wäre. Somit kennt man die Risikoverminderung, welche durch die Schliessung des Passes an diesem Tag erreicht werden konnte. Die Risikoverminderung kann als Wirksamkeit dieses Sperrtages bezeichnet werden. In einem nächsten Schritt werden die Sperrtage pro Winter so verteilt, dass sie in abnehmender Reihenfolge möglichst viele Lawinenabgänge pro Tag abgedeckt respektive das Risiko möglichst stark vermindert hätten. Damit kann die Risikoverminderungsfunktion pro Winter grafisch dargestellt werden (Abbildung 7). Die Kurve zeigt auf, mit welcher Anzahl Sperrtage welche Risikoverminderung hätte erreicht werden können, wenn die Sperrtage optimal gewählt worden wären und die Strasse nur an denjenigen Tagen gesperrt worden wäre, an welchen auch tatsächlich Strassenverschüttungen stattfanden. Der Durchschnitt der untersuchten zehn Jahre stellt die theoretisch optimale Risikoverminderungsfunktion dar.

Herleitung der Risikoverminderungsfunktion mit Massnahmen und Bestimmung des Restrisikos Gemäss Wilhelm (1999) kann das Restrisiko für die Strassenbenützer, welches mit einer gewählten Massnahme respektive einer Massnahmenkombination bestanden hat, rückblickend abgeschätzt werden. Das Verhältnis der gesamten Anzahl durch spontane Lawinen in einem Winter verursachter Strassenverschüttungen zur Anzahl Strassenverschüttungen bei offener Strasse bestimmt im Wesentlichen

\begin{tabular}{|c|c|c|c|}
\hline Merkmai $x^{2}$ & $\begin{array}{l}\text { Rishoanilyse 1998 } \\
(S L \text { f }\end{array}$ & $\begin{array}{l}\text { Risisoanalyse 2011 } \\
\text { Conceprio \& Gasser 2011) }\end{array}$ & Bemerkungen, \\
\hline $\begin{array}{l}\text { Anzahil Lawinenabgange } \\
\text { wăhrend zehn Jahren }\end{array}$ & 340 & $\begin{array}{l}\text { 338, wovon } 159 \text { kunstlich ausge- } \\
\text { lost und } 179 \text { spontan abgegangen }\end{array}$ & Untersuchungsperiode $2000-2010$ \\
\hline Wiederkehrdauer der l lawinen & Katasterdaten, eiganzt mit & $\begin{array}{l}\text { Neuteut teilung mit den detalle } \\
\text { Terten baten von } 2000 \text { bis } 2010\end{array}$ & $\begin{array}{l}\text { Que Wieder kehrdauein wurden } 2017 \\
\text { generell etwas erhoht }\end{array}$ \\
\hline $\begin{array}{l}\text { Mittlere Lawinenbreiten in } \\
\text { Bereich der Strasse }(m)\end{array}$ & $\begin{array}{l}\text { Rudimentare Katasterdaten, er } \\
\text { ganzt mit Schätzungen in Feld }\end{array}$ & $\begin{array}{l}\text { Neubeurteilung mit den detail. } \\
\text { lierten Daten von } 2000 \text { bis } 2010\end{array}$ & $\begin{array}{l}\text { Dile Lawinenbreiten wurden } 2017 \text { um rund } \\
28 \% \text { vermindert. }\end{array}$ \\
\hline 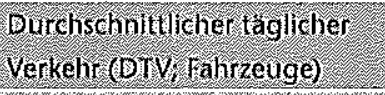 & 1000 & $\begin{array}{l}\text { Effektiv } 409 \text {, } \\
\text { gerechnet mit } 500\end{array}$ & 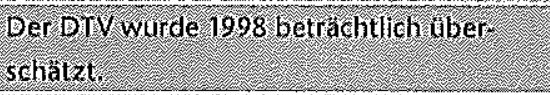 \\
\hline $\begin{array}{l}\text { Mittlerer Besetzungsgrad } \\
\text { (B) Personen/Fahrzeug) }\end{array}$ & 1.6 & 2.0 & 2017 erholt aufgrund passantenbefragung \\
\hline $\begin{array}{l}\text { Koilektives Ausgangsis ko } \\
\text { (todestalletlahr) }\end{array}$ & 0.51 & 0,167 & $\begin{array}{l}\text { Die Differenz st weitgehend mit dem DV V } \\
\text { e elilarbar }\end{array}$ \\
\hline $\begin{array}{l}\text { Individuelles Todesfallisiko } \\
\text { der Sicherungsdienste ( } / \text { /labir) }\end{array}$ & $6.3 \times 10^{-4}$ & $9.0 \times 10-1$ & $\begin{array}{l}\text { Die Different ist mit der fahrzeugge } \\
\text { schwindigkeit erklarbar. }\end{array}$ \\
\hline Riskospitzen (Todesfalle/Jh) & Nicht quantifizien & Bis zu 5 faches kollektives Risiko & Separat berechnet und gual tativ beurteilt \\
\hline
\end{tabular}

Tab 2 Daten und Resultate (Ausgangsrisiken, d.h. ohne Massnahmen) der Risikoanalysen von 1998 und 2011 im Vergleich. 


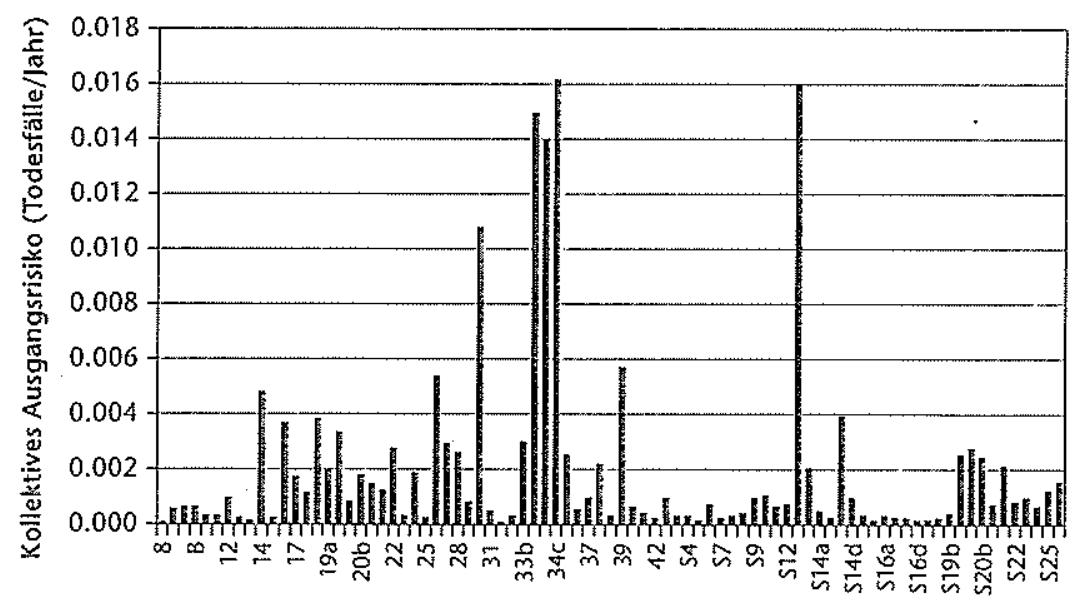

Lawinenzüge

Abb 6 Zusammenstellung des kollektiven Ausgongsrisikos (ohne Sperrungen und künstliche Lawinenauslösung) für ausgewählte Lawinenzüge. Sehr gut ersichtlich ist die Häufung von Lawinenzügen mit mittlerem bis grossem Risiko im Bereich des Lawinenzugs Nr. 34.

das Restrísiko. Dieses Verhältnis und somit auch das Restrisiko konnte direkt aus den verfügbaren Lawinendaten der Winter 2000/2001 bis 2009/2010 be. rechnet werden. Anlrand der Anzahl Sperrtage, welche tatsächlich realisiert wurden, kann die theoretisch optimale Risikoverminderungsfunktion so extrapoliert werden, dass sie die tatsächliche Risikoverminderungsfunktion für die gewählte Massnahmenkombination darstellt.

\section{Resultate}

Vergleich der Ausgangsrisiken von 1998 und 2011

Nachfolgend werden die im Jahr 1998 (SLF 1998) und 2011 (Conceprio \& Gasser 2011) berechneten Ausgangsrisiken analysiert (Tabelle 2). 1998 mussten die Wiederkehrdauern und Verschüttungsbreiten wegen mehrheitlich fehlender Katasterangaben gutachterlich abgeschätzt werden. 2011 standen die Aufzeichnungen von 338 Lawinenabgängen auf die Strasse zur Verfügung, wovon 159 künstlich ausgelöst wurden, 179 natürlich abgegangen sind und 7 die offene Strasse verschütteten. 1998 wurden die Wiederkehrdauern etwas unter- und die mittleren Verschüttungsbreiten der Lawinen etwas überschätzt. Die 1998 für eine Periode von zehn Jahren abgeschätzten 340 Lawinen stimmen praktisch mit der tatsächlichen Anzahl Beobachtungen überein.

Der durchschnittliche tägliche Verkehr (DTV) wurde 1998 mit 1000 Fahrzeugen im Vergleich mit dem in den letzten zehn Jahren erfassten DTV von 409 Fahrzeugen überschätzt. Die Neuberechnung des Risikos wurde schliesslich mit einem DTV von 500 Fahrzeugen durchgeführt, da aufgrund des leicht ansteigenden Trends davon ausgegangen wird, dass der DTV in den kommenden Jahren etwas zunehmen dürfte.

Für die Fahrzeuggeschwindigkeiten und die Letalität wurden in beiden Studien die gleichen Werte angenommen (Geschwindigkeit: differenziert nach Streckenabschnitt; Letalität $\lambda=0.4$ ). Für die Bcstimmung des individuellen Todesfallrisikos der Sicherungs- und Schneeräumungsequipen wurden 2011 die Fahrzeuggeschwindigkeiten mit durchgehend $40 \mathrm{~km} / \mathrm{h}$ etwas geringer eingesetzt.

Für das kollektive Ausgangsrisiko resultiert in der Risikobeurteilung von 2011 mit 0.167 Todesfällen pro Jahr ein deutlich geringeres kollektives Ausgangsrisiko, als 1998 mit 0.51 Todesfällen pro Jahr berechnet wurde. Die Differenz beruht hauptsächlich auf dem $1998 \mathrm{zu}$ hoch angenommenen DTV. Da auch alle künstlich ausgelösten Lawinen zur Berech nung beigezogen wurden, wird das Ausgangsrisiko 2011 eher überschätzt. Das 2011 ermittelte kollektive Todesfallrisiko ohne Massnahmen mit durchschnittlich 1.67 Todesfällen wäbrend zehn Jahren wäre nicht akzeptabel.

Für das individuelle, jährliche Todesfallrisiko der Sicherungsdienste resultiert $2011 \mathrm{mit} 9 \times 10^{-4}$ ein Ieicht höherer Wert als 1998 mit $6.3 \times 10^{-4}$, was mit den 2011 reduzierten Geschwindigkeiten für Sicherungsdienste erklärbar ist. Der Unterschied liegt allerdings im Bereich der Unsicherheiten solcher Risikoabschätzungen.

Zusammenfassend kann man festhalten, dass die Risikoanalysen von 1998 und 2011 sehr ähnliche Resultate liefern. Dies zeigt, dass auch auf lückenhaften Daten beruhende Risikoanalysen zu vertrauenswürdigen Resultaten führen können, wenn die Abschätzungen sorgfältig durchgeführt werden.

Die 84 Lawinenzüge weisen sehr unterschiedliche Wiederkehrdauern und Verschüttungsbreiten auf und verursachen somit sehr unterschiedliche Ausgangsrisiken (Abbildung 6). So sind die fünf Lawinenzüge Nr. 30 (Stgegia), Nr. 34a-c (nördlich ScopiGalerie) und Nr. S13 (Acquacalda) für gut $43 \%$ des Ausgangsrisikos verantwortlich. Die räumliche $\mathrm{Ab}-$ folge der Lawinenzüge mit grossen Risiken ist vor allem im Hinblick auf mögliche Risikospitzen von Bedeutung. Am Lukmanierpass sind zwei Situationen wesentlich, welche zu Risikospitzen führen können: 1) Verkehrsspitzen (z.B. um die Ostertage besteht ein rund fünfmal grösserer DTV und demzufolge ein rund fünfmal grösseres Risiko);

2) stehender Verkehr im Bereich von nahe beieinander liegenden Lawinenzügen mit hohen Risiken (wenn eine erste Lawine die Strasse verschüttet und die Fahrzeuge sich auf beiden Seiten stauen, ist der stehende Verkehr durch weitere Lawinenabgänge stark gefährdet). Diese Situation bewirkt im Bereich des Lawinenzuges 34 (nördlich der Scopi-Galerie) ein bis zu fünffaches Risiko.

\section{Restrisiken mit heutigem Sicherungskonzept}

Um das heute noch bestehende Restrisiko einschätzen zu können, muss die Wirkung des angewendeten Sicherungskonzepts quantitativ analysiert werden. In der Studie von 1998 (SLF 1998) wurden 


\begin{tabular}{|c|c|c|c|}
\hline Mrnmal) & 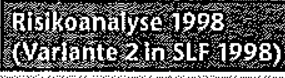 & 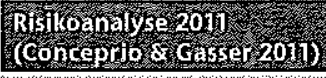 & 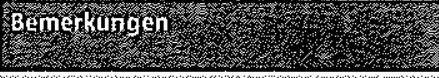 \\
\hline $\begin{array}{l}\text { Erforderliche Anzahl Sperrlage } \\
\text { fur weitgehende } \\
\text { Risikoverminderung }\end{array}$ & $70(40,100)$ & 71 & $\begin{array}{l}\text { Schätzung von } 1998 \text { wird trotz } \\
\text { grossen streuungen mit auswer } \\
\text { tungen bestatigt. }\end{array}$ \\
\hline $\begin{array}{l}\text { Ertorderliche Anzahl Speritage } \\
\text { nit kunsulicter Lawinenauslosung }\end{array}$ & $28(13-42)$ & 43 & 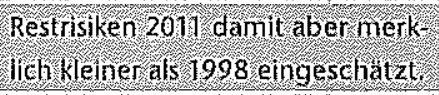 \\
\hline $\begin{array}{l}\text { Kollektives Restrisiko } \\
(\% \text { des Ausgangsrisikos) }\end{array}$ & $26(23-29)$ & 4 & $\begin{array}{l}\text { Sicherungsdienst hat besser } \\
\text { gearbeitet als } 1998 \text { erwartet. }\end{array}$ \\
\hline $\begin{array}{l}\text { Andividuelles Todesfallerestrisiko } \\
\text { der Stcherungsdienste (WJahr) }\end{array}$ & $1.64 \times 101$ & $200 \times 10$ & $\begin{array}{l}\text { Individuelle Risiken sind } 2011 \text { in } \\
\text { akzeptablen Bereich }\end{array}$ \\
\hline
\end{tabular}

Tab 3 Daten der Risikoanalysen von 1998 und 2011 zur Abschötzung der Wirkung des Sicherungskonzepts und zur Berechnung der kollektiven und individuellen Restrisiken im Vergleich.

fünf Varianten von Sicherungsmassnahmen untersucht. Am besten vergleichbar mit der heutigen Situation ist die Variante 2 mit Strassensperrung und künstlicher Lawinenauslösung (SLF 1998). Dieser Vergleich liegt der Tabelle 3 zugrunde.

Wegen fehlender Daten wurde 1998 (SLF 1998) die erforderliche Anzahl Sperrtage für eine «weitgehende Risikoverminderung" anhand der Gefahrenstufen des Lawinenbulletins (SLF 2013) abgeschätzt. Dazu wurden alle Tage mit der Gefahrenstufe grösser oder gleich "erheblich" und 50\% der Tage mit Stufe «mässig» berücksichtigt, was 70 Sperrtage und Restrisiken von rund $13 \%$ ergab. Die Auswertung des lokalen Lawinenbulletins der Pro Lucmagn von $2002 / 2003$ bis $2009 / 2010$ ergibt mit 71 Tagen eine sehr gute Übereinstimmung.

Die erforderliche Anzahl Sperrtage mit künstlicher Lawinenauslösung wurde in Variante 2 (SLF 1998) mit 28 Tagen (13-42 Tage) und einem Restrisiko von $26 \%(23-29 \%)$ grob eingegrenzt. Mit dem angewendeten Sicherungskonzept ergaben sich 43 Sperrtage, wobei während 10 Jahren noch 7 La-

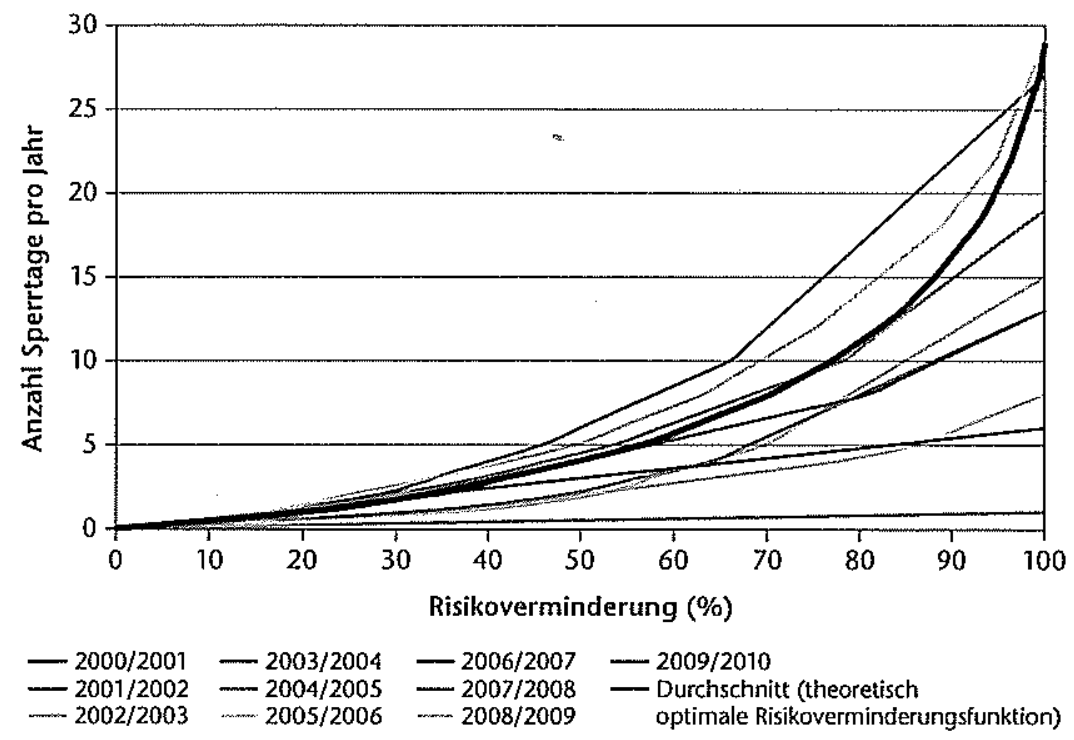

$A b b 7$ Theoretisch optimale Risikoverminderung mit Sperrtagen. Auswertung der Lawinendaten von 2000 bis 2010 zur optimalen Reihung der minimal erforderlichen Sperrtage (in Anlehnung an Wilhelm 1999). wịnen auf die geöffnete Strasse abgegangen sind. Das heute noch bestehende Restrisiko berechnet sich mit 7 Lawinen dividiert durch 179 natürlich abgegangene Lawinen zu $4 \%$. Das defensive Vorgehen bei der Strassenöffnung hat sicher zu den geringen Restrisiken beigetragen. Zu erwähnen ist, dass die $7 \mathrm{La}-$ winen bei durchschnittlich 97 Öffnungstagen (181 minus 84 ) pro Winter abgegangen sind. Würden nur die effektiven Lawinensperrtage von 43 Tagen berücksichtigt, so wären an 138 Öffnungstagen (181 minus 43) etwas mehr als 7 Lawinen zu erwarten gewesen. Das Restrisiko ist somit mit $4 \%$ sicher optimistisch eingeschätzt. Die individuellen Restrisiken der Sicherungsdienste sind mit $3.6 \times 10^{-5}$ Todesfällen pro Jahr in einem akzeptablen Bereich (in Bründl 2009 wird für Schneeräumungsequipen ein Grenzwert von $1 \times 10^{-4}$ vorgeschlagen).

\section{Beurteilung der Wirkung der Strassensperrungen}

Wenn spontane Lawinen abgehen, sollte die Strasse gesperrt sein. Je besser dies gelingt, umso höher ist die Wirkung der Strassensperrungen. Die nachfolgende Auswertung nach Wilhelm (1999) hilft, dies zu beurteilen. Die Abbildung 7 zeigt die Sperrtage in abnehmender Wirksamkeit als Kurvenschar für zehn Winter. Die erforderlichen Sperrtage pro Winter streuen sehr stark. Im lawinenärmsten Winter 2001/2002 wäre kein einziger Sperrtag nötig gewesen für eine 100-prozentige Risikoverminderung, im lawinenreichsten Winter 2000/2001 hätte mit zehn Sperrtagen hingegen lediglich eine Risikoreduktion von $66 \%$ erreicht werden können.

Der Durchschnitt über die zehn Jahre stellt die theoretisch optimale Risikoverminderungsfunktion dar (Abbildung 7). Wenn die Strasse also nur an denjenigen Tagen gesperrt worden wäre, an welchen tatsächlich Lawinen auf die Strasse niedergingen, hätten durchschnittlich 29 Sperrtage pro Winter ausgereicht, um das Risiko von Strassenverschüttungen komplett zu eliminieren. Die «besten» vier Sperrtage hätten das Risiko im Durschnitt bereits auf $50 \%$ vermindert, während die Risikoverminderung von 50 auf $100 \%$ die restlichen 25 Sperrtage erfordert hätte. 


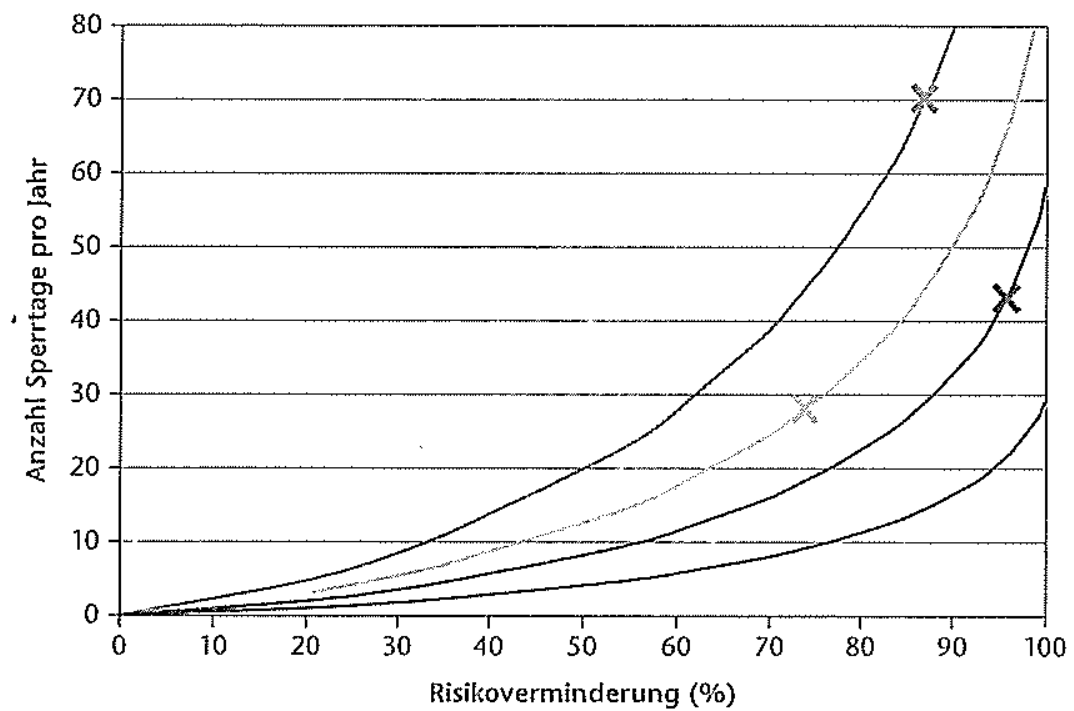

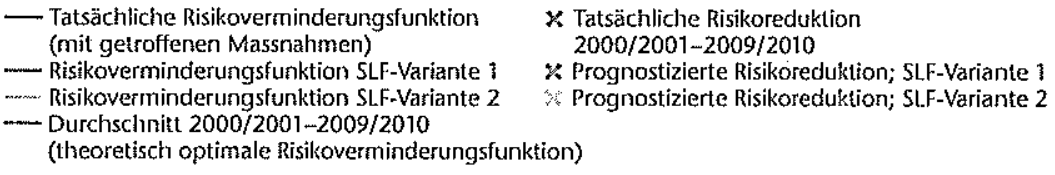

Abb 8 Mit den getroffenen Massnahmen «Sperren (Südseite) und Sperren/Sprengen (Nordseite)" wurde mit durchschnittlich 43 Sperrtagen eine Risikoverminderung von $96 \%$ erreicht. Zum Vergleich sind die Varianten 1 und 2 aus SLF (1998) aufgetragen, die bei gleicher Risikoverminderung mehr Sperrtoge erfordert hätten.

Mit dem heutigen Sicherungskonzept sind mit 43 Sperrtagen noch Restrisiken von $4 \%$ vorhanden, d.h., die Risikoverminderung beträgt $96 \%$. Extrapoliert man nun die theoretisch optimale Risikoverminderungsfunktion aus der Abbildung 7 durch den Punkt «43 Sperrtage/Risikoverminderung 96\%», erhält man die tatsächliche Risikoverminderungsfunktion für das heutige Sicherungskonzept (Abbildung 8). Daraus wird ersichtlich, dass durchschnittlich 52 Sperrtage nötig wären, um das Risiko von Strassenverschüttungen um $100 \%$ zu vermindern; mit durchschnittlich lediglich 8 sperrtagen kann das Risiko heute durchschnittlich bereits um die Hälfte reduziert werden.

Das Verhältnis der tatsächlichen Risikoverminderungsfunktion zur theoretisch optimalen $\mathrm{Ri}$ sikoverminderungsfunktion beträgt 1.8 (52/29), was im Vergleich zum Flüelapass mit 4.4 (Wilhelm 1999) einen sehr guten Wert darstellt. Das heisst mit andern Worten, dass der Sicherungsdienst beim Lukmanierpass gegenüber der theoretisch optimalen Risikoverminderung (im Nachhinein mit allen Lawinendaten berechnet) bei seiner Entscheidung nur 1.8 MaI mehr Sperrtage benötigt hat.

Zum Vergleich sind in Abbildung 8 die Risikoverminderungsfunktionen für die Varianten 1 (nur Sperren) und 2 (Sperren und Lawinensprengung) des SLF (1998) eingetragen. Variante 1 würrde für eine weitgehende Risikoverminderung (Restrisiko $13 \%$ ) durchschnittlich 70 Sperrtage erfordern. Die Variante 2 ist gekennzeichnet mit 28 Sperrtagen und einem Restrisiko von $26 \%$. Beide Risikovermin- derungsfunktionen hätten mehr Sperrtage erfordert, als der Sicherungsdienst vom Lukmanierpass für die gleiche Risikoverminderung effektiv einsetzen musste.

\section{Schlussfolgerungen und Ausblick}

Für die Risikoanalyse standen 1998 sehr wenige Daten zur Verfügung, und es mussten verschiedene Faktoren abgeschätzt werden. Im Nachhinein wurde festgestellt, dass insbesondere aufgrund des damals überschätzten DTV die Risiken etwas überschätzt wurden. Insbesondere die gutachterliche Einschätzung der Wiederkehrdauer der Lawinen auf der Südseite, für welche praktisch keine Aufzeichnungen vorhanden waren, kann aus heutiger Sicht als sehr realistisch bezeichnet werden.

Die Wirkung des Sicherungskonzeptes der Passstrasse hängl entscheidend von der Qualität der Entscheidungen des Sicherungsdienstes ab. Die geringe Anzahl von Lawinenabgängen auf die geöffnete Strasse weist darauf hin, dass die Mehrheit der Sperrungen zum richtigen Zeitpunkt erfolgte. Die hohe Wirksamkeit der Sperrtage zeigt, dass nicht notwendige Strassensperrungen selten waren. Trotzdem muss gesagt werden, dass die starke Abhängigkeit des Sicherungskonzepts vom Faktor Mensch wahrscheinlich das grösste Risiko darstellt; Fehler des Sicherungsdienstes respektive «falsche» Entscheide können fatale Folgen haben. Zu Beginn des Projektes Winteröffnung Lukmanierpass war nicht klar, ob nur mit den organisatorischen Massnahmen Sperren und künstliche Lawinenauslösung eine genügende Risikoverminderung erreicht werden kann. Die Tatsache, dass die Schliesstage unfallfrei von im Durchschnitt 155 auf 37 gesenkt werden konnten, zeigt, dass eine Winteröffnung des Lukmanierpasses mit bloss organisatorischen Massnahmen vertretbar war. Dabei muss aber berücksichtigt werden, dass der heutige Verkehr bedeutend geringer ist, als ursprünglich angenommen wurde.

Die Verfügbarkeit der Passstrasse hängt mit dem gewählten Sicherheitskonzept stark von den jeweiligen Witterungsverhältnissen ab: Im «besten» Winter blieb der Pass gerade einmal vier Tage ganz geschlossen, im «schlechtesten» hingegen mehr als 100. Dies ist zwar bezüglich der Sicherheit kein Problem, die inkonstante Verfügbarkeit ist aber wohl ein grosser Unsicherheitsfaktor für die Strassennutzer, was sich auch auf das Verkehrsaufkommen auswirkt.

Je mehr der Pass geöffnet ist, desto mehr Verkehr wird generiert. Diese Hypothese des Vereins Pro Lucmagn wurde bestätigt. Der DTV scheint hingegen mit zunehmender Verfügbarkeit nicht zuzunehmen. Ein Grund dafür dürften die starke Wetterabhängigkeit und die damit verbundenen Unsicherheiten der 
Strassenöffnung sein. Weitere Gründe wie regionale Konkurrenzsituation im Tourismus dürften für das Verkehrsaufkommen ebenfalls eine Rolle spielen. Der Verein Pro Lucmagn strebt eine Erhöhung der Verfügbarkeit der Passstrasse im Winter an. Ein erhöhtes Verkehrsaufkommen führt auch zu höheren Risiken. Wird ein solches tatsächlich erreicht, muss allenfalls auch das Sicherungskonzept angepasst werden.

Aus heutiger Sicht gelangt die Umsetzung einer Winteröffnung des Lukmanierpasses «nur» mit organisatorischen Massnahmen an Grenzen. Es scheint kaum noch Spielraum zu geben, um bei gleichbleibender Sỉcherheit die Verfügbarkeit der Passstrasse erhöhen zu können.

Wie eine zweite von den Kantonen Tessin und Graubünden in Auftrag gegebene Studie zur Evaluation möglicher baulicher Massnahmen im Jahr 2013 zeigte (Conceprio \& Gasser 2013), gibt es allenfalls einige kleinere Möglichkeiten für bauliche Massnahmen zur Verbesserung der Bewirtschaftung (d.h. weniger Aufwand bei der Räumung, Vereinfachung der Arbeitsabläufe insbesondere bei der Lawinensprengung). Um massgebliche Verbesserungen bezüglich der Sicherheit und Verfügbarkeit der Passstrasse zu erzielen, wären allerdings kostspielige Galerien notwendig.

Eingereicht: 10. Dezember 2013, okzeptierl (mit Review): 22. Mai 2014

\section{Dank}

Den Verantwortlichen der Kantone Tessin und Graubünden sowie der Pro Lucmagn wird herzlich gedankt für die gute Zusammenarbeit und die Bereitstellung der Daten.

\section{Winteröffnung des Lukmanierpasses mit organisatorischen Massnahmen}

Der Lukmanierpass wurde seit dem Winter 2000/2001 während zehn Wintern durch den Verein Pro Lucmagn versuchs. weise offen gehalten. Das Gesuch um Erteilung einer unbefristeten Bewilligung im Jahr 2010 gab den Ausschlag zu einer Überprüfung des Sicherungskonzeptes und zur Neubeurtejlung des Risikos. Insgesamt zeigt sich, dass mit den gewählten Massnahmen "Strassensperrung und Lawinensprengung" eine grössere Reduktion der Risiken erreicht werden kann als ursprünglich angenommen. Die abgeschätzten Restrisiken liegen in einem akzeptablen Bereich. Trotzdem verbleiben mit den gewählten organisatorischen Massnahmen erhebliche Risiken, die hauptsächlich mit dem Unsicherheitsfaktor «menschliche Beurteilung» zusammenhängen. Rückblickend zeigt sich, dass die Risikoanalyse aus dem Jahr 1998 sehr geeignet war, den Versuchsbetrieb der Winteröffnung am Lukmanierpass im Voraus zu beurteilen.

\section{Literatur}

ASTRA (2012) Naturgefahren auf den Nationalstrassen: Risikokonzept. Ausgabe 2012 (V2.10). Bern: Bundesamt Strassen, Dokumentation ASTRA 89001. $96 \mathrm{p}$.

BRÜNDL M (2009) Risikokonzept für Naturgefahren - Leitfaden. Bern: Nationale Plattform Naturgefahren. 420 p.

BUWAL (1999) Risikoanalyse bei gravitativen Naturgefahren - Methode. Bern: Bundesamt Umwelt Wald Landschaft, UmweltMaterialien 107/I. $117 \mathrm{p}$.

CONCEPRIO F, GASSER N (2011) Aktualisierung des SLF-Gutachtens 98.01 und Neubeurteilung des Risikos. Dongio: LucchiniMariotta Associati SA, unveröffentlicht.

CONCEPRIO F, GASSER N (2013) Massnahmenevaluation zur verbesserung der Passbewirtschaftung im Winter. Dongio: Lucchinì-Mariotta Associati SA, unveröffentlicht.

MARGRETH S, STOFFEL L, WILHELM C (2003) Winter opening of high alpine pass roads - analysis and case studies from the Swiss Alps. Cold Reg Sci Technol 37: 467-482.

PLANAT (1998) Von der Gefahrenabwehr zur Risikokuitur - PLANAT, die nationale Plattform Naturgefahren stellt sich vor. Bern: Nationale Plattform Naturgefahren. $19 \mathrm{p}$.

RHEINBERGER CM, BRÜNDL M, RHYNER J (2009) Dealing with the White Death: avalanche risk management for traffic routes. Risk Anal 29: 76-94.

SLF (1998) Offenhaltung der Lukmanierstrasse im Winter. Davos: Eidgenöss institut Schnee-Lawinenforschung, SLF Gutachten G98.01, unveröffentlicht.

SLF (2000) Der Lawinenwinter 1999. Ereignisanalyse. Davos: Eidgenöss Institut Schnee- Lawinenforschung. $588 \mathrm{p}$.

SLF (2013) Lawinenbulletins und weitere Produkte. Interpretationshilfe. Davos: WSL-Institut Schnee- Lawinenforschung, $13 \mathrm{ed} .51 \mathrm{p}$.

STOFFEL L. (2001) Künstiliche Lawinenauslösung. Praxishilfe. Davos: Eidgenöss Institut Schnee- Lawinenforschung, Mitt 53.66 p. WILHELM C (1999) Kosten-Wirksamkeit von Lawinenschutz-Massnahmen an Verkehrsachsen. Vorgehen, Beispiele und Grundlagen der Projektevaluation. Bern: Bundesamt Umwelt Wald Landschaft, Praxishilfe. $110 \mathrm{p}$.

\section{Ouverture hivernale avec mesures organisationnelles du col du Lukmanier}

Le col du Lukmanier a fait l'objet d'un essai par l'association Pro Lucmagn d'ouverture hivernale depuis l'hiver 2000/2001 pendant dix ans. La demande en 2010 pour l'octroi d'une autorisation à durée illimitée a conduit à la révision du concept de sécurité et à la réévaluation du risque. Dans l'ensemble, les mesures pratiquées «fermeture des routes et déclenchement artificiel d'avalanches" ont permis d'atteindre une plus grande réduction du risque qu'admis jusqu'à présent. Les risques résiduels estimés sont tolérables. Malgré tout, avec les mesures choisies, il reste des risques conséquents qui sont dus principalement au facteur d'incertitude lié à l'«appréciation humaine». Avec du recul, on peut constater que I'analyse de risque de 1998 était très appropriée pour juger à l'avance de l'essai-pilote d'ouverture hivernale du col du Lukmanier. 